\title{
Thermal Lie Groups, Classical Mechanics, and Thermofield Dynamics
}

\author{
Ademir E. Santana* and F. C. Khanna \\ Department of Physics, University of Alberta, Edmonton, Alberta T6G 2J1, Canada; and \\ TRIUMF, 4004, Wesbrook Mall, Vancouver, British Columbia V6T 2A3, Canada
}

\author{
AND \\ H. Chu AND Y. C. Chang \\ Department of Physics, University of Illinois, Urbana, Illinois 61801
}

Received September 20, 1995

\begin{abstract}
The concept of thermoalgebra, a kind of representation for the Lie-symmetries developed in connection with thermal quantum field theory, is extended to study unitary representations of the Galilei group for thermal classical systems. One of the representations results in the first-quantized Schönberg formalism for the classical statistical mechanics. Furthermore, the close analogy between thermal classical mechanics and thermal quantum field theory is analysed, and such an analogy is almost exact for harmonic oscillator systems. The other unitary representation studied results in a field-operator version of the Schönberg approach. As a consequence, in this case the counterpart of the thermofield dynamics (TFD) in classical theory is identified as both the first and second-quantized form of the Liouville equation. Non-unitary representations are also studied, being, in this case, the Lie product of the thermoalgebra identified as the Poisson brackets. A representation of the thermal $S U(1,1)$ is analysed, such that the tilde variables (introduced in TFD) are functions in a double phase space. As a result the equations of motion for dissipative classical oscillators are derived.

(c) 1996 Academic Press, Inc.
\end{abstract}

\section{INTRODUCTION}

The main goal of this paper is to study representations of symmetry groups associated with thermal classical systems [1]. To do this we utilize the basic elements of the representation theory of Lie groups developed in the context of pure state representation of the thermal quantum field theory.

In quantum field theory we can double the degrees of freedom of a system so that a finite temperature mixed state can be represented by a pure state $[2,3]$. The

* On leave from the Instituto de Fisica, Universidade Federal da Bahia, Campus de Ondina, 40210-340, Salvador, Bahia, Brazil. E-mail address: santana@phys.ualberta.ca. 
doubling process, called tilde conjugation rules, is related with an algebraic association among the generators of Lie symmetries and dynamical observables through the concept of thermoalgebra [4]. Moreover, the temperature effects arise via vacuum correlations between the so called tilde and non-tilde variables. The explicit formalism of such a theory is known as the thermofield dynamics (TFD) [5-8] and has been fully developed in recent years. It can be used to treat both equilibrium and non-equilibrium systems and has shown its advantages to other methods, among which the convenient diagram recipes and the time dependent Bogoliubov transformations are the most celebrated.

Nevertheless, an extension of the doubling process to classical systems cannot be carried out in a straightforward manner. This is because two mutually non-interacting systems can be related with each other through vacuum correlations in a quantum model, while this is not the case for the classical theory. The counterpart of TFD in classical mechanics is yet to be identified, and this is accomplished in this paper through the analysis of representations of thermal Lie algebras.

The concept of thermoalgebra has been successfully used to analyse representations of thermal quantum systems described by the Galilei and Poincare invariance $[4,9]$. Here we show that such an approach can be extended to thermal classical theory as well. It means that, we can apply the techniques developed in TFD to the study of thermal classical systems. In addition, the classical analogues to some elements of the thermal field theories are specified. Such an achievement, in particular, sheds some light on the true physical nature of certain elements of TFD, which have not been fully interpreted and appreciated physically and are brought forth by the introduction of the dual (or tilde) operators.

In order to study representations of Lie groups for thermal classical systems, first, we search for unitary representations of thermal Lie algebras. In this case, the Lie product of the thermoalgebra is the commutator, and the inner product in the representation space is defined on a set of complex functions in the phase space. Only in a particular case such functions are assumed to be of the type $L^{2}$ (Lebesgue integral). Second, a non-unitary representations for a classical system by using the structure of the Poisson brackets as the Lie product of the thermoalgebra is introduced.

Unitary representations of Lie algebras for classical systems have been studied in the literature in different ways [10-17]. In particular, Loinger [11] has derived unitary representations for the Galilei group associated with the Liouville equation, starting from the structure of the adjoint representations of the usual classical mechanics [18].

In contrast to the usual methods, however, we show that the thermoalgebraic structure provides a form to analyse the Galilei group for classical systems without relying on a particular unitary representation. That is, the role played by each element of the thermoalgebra, as well as its commutation relations, is defined by physical and algebraic aspects, without referring to the adjoint representation of the classical mechanics. In this context, the phase space structure arises naturally, and the Schönberg-Liouville wave equation is derived $[19,20]$. Another unitary 
representation gives the Schönberg-Liouville equation in terms of field operators defined on a Fock space of symmetrical and antisymmetrical wave functions of classical particles $[19,21-23]$. In both situations, it is clear that the basic elements of the TFD can be used to describe classical systems; and consequently, the counterpart of the TFD is identified as the Schöberg formalism for the classical statistical mechanics.

As a particular example, we concentrate on the structure of the classical harmonic oscillator system, which can be mapped on to two sets of mutually commuting oscillators. For such a model, the analogy with TFD is almost exact. The original Hamiltonian, which commutes with the time evolution generator, can be mapped to the Bogoliubov-transformation generators in TFD, which introduces temperature effects. Thus the classical system exhibits some quantum-like elements at finite temperature.

In the case of non-unitary representations, the thermoalgebra of the $S U(1,1)$ group is studied. Using a particular class of generators of time translations (a class of hat-Hamiltonians), we derive the equations for dissipative classical harmonic oscillators which were proposed by Feshbach and Tikochinsky [24], and recently studied in connection with quantum optics and thermal field theories [25-27]. The nature of tilde variables are, then, written explicitly as functions defined on a double phase space.

The paper is organized in the following way. In Section 2 the basic elements defining the concept of classical representations for a thermal Lie algebra are introduced. In Section 3 the classical unitary representations for the thermal Galilei group are studied resulting in the Schönberg approach. In Section 4 the Schönberg-Liouville wave equation for the harmonic oscillator is discussed in connection with the TFD formalism. The Fock space version for the classical unitary representations is analysed in Section 5; the representations of $S U(1,1)$ group on the double phase space are discussed in Section 6. Finally, in Section 7 some final remarks and conclusions are presented.

\section{REPRESENTATIONS OF THERMAL LIE ALGEBRAS FOR CLASSICAL SYSTEMS}

In thermal quantum field theory, the thermal Lie algebra concept is defined in the following way. Let $\mathscr{L}=\left\{A_{i}, \mid A_{i} \diamond A_{j}=C_{i j k} A_{k}\right\}$ be the Lie algebra associated with the usual dynamical physical variables, where $\diamond$ denotes the Lie product, and $C_{i j k}$ are the structure constants. We then associate this algebra with an isomorphic hat-algebra denoted by $\hat{\mathscr{L}}=\left\{\hat{A}_{i}, \mid \hat{A}_{i} \diamond \hat{A}_{j}=C_{i j k} \hat{A}_{k}\right\}$, such that the thermoalgebra, designated by $\mathscr{L}_{T}$, is defined by [4]

$$
\begin{gathered}
A_{i} \diamond A_{j}=C_{i j k} A_{k}, \\
A_{i} \diamond \hat{A}_{j}=C_{i j k} A_{k}, \\
\hat{A}_{i} \diamond \hat{A}_{j}=C_{i j k} \hat{A}_{k} .
\end{gathered}
$$


In TFD, given the tilde operators $\tilde{A}$ that is isomorphic to the non-tilde operator $A$ through the doubling process, the hat operators may be identified as $\hat{A}=A-\tilde{A}$. In general, however, such an association should not be assumed. Instead, the search for the hat operators should be our primary goal.

The main characteristics of $\mathscr{L}_{T}$ are the following: (i) As a vector space, $\mathscr{L}_{T}$ is the direct sum of $\mathscr{L}$ and $\hat{\mathscr{L}}$. (ii) $\mathscr{L}$ is an ideal of $\mathscr{L}_{T}$. (iii) From the dynamical standpoint, the elements of the subalgebra $\hat{\mathscr{L}}$ are interpreted as dynamical generators of symmetries and the elements of $\mathscr{L}$ are the usual dynamical observables. Both are called dynamical variables. In the Lie algebra approach, infinitesimal transformations of dynamical variables are induced by the (Lie) product of the generators. Then Eq. (2) dictates just how the dynamical generators act on the dynamical variables infinitesimally. (iv) Eq. (1) defines the non-abelian nature of the dynamical observables, related to the measurement processes.

The algebra $\mathscr{L}_{T}$ can be derived from Eq. (1) in association with the concept of tilde conjugation rules and the formalism of the Lie-Baxter algebras [4]. This way of deriving the thermoalgebra $\mathscr{L}_{T}$, however, is not convenient if the observable algebra is an abelian algebra, as it is the case when we search for unitary operators for classical systems. Nevertheless, in such a situation we can still define the concept of thermoalgebra for classical systems, since property (iv) can be weakened by writing Eq. (1) as $A_{i} \diamond A_{j} \equiv\left[A_{i}, A_{j}\right]=0$, where $[A, B]=A B-B A$, the commutator, is the Lie product. Notice that in this case we still have the basic properties (i)-(iii), but property (iv) should be generalized to include abelian observables. Then we assume that a unitary thermoalgebra describing a classical system should be given by

$$
\begin{aligned}
& {\left[A_{i}, A_{j}\right]=0,} \\
& {\left[A_{i}, \hat{A}_{j}\right]=i C_{i j k} A_{k},} \\
& {\left[\hat{A}_{i}, \hat{A}_{j}\right]=i C_{i j k} \hat{A}_{k} .}
\end{aligned}
$$

In this case, the hat operators are a faithful representation of the symmetry operations.

On the other hand, one way to maintain the properties (i) to (iv) for abelian observables is to define the Lie product of the thermoalgebra as the Poisson brackets. In this case we can write

$$
\begin{aligned}
& \left\{A_{i}, A_{j}\right\}=C_{i j k} A_{k}, \\
& \left\{A_{i}, \hat{A}_{j}\right\}=C_{i j k} A_{k}, \\
& \left\{\hat{A}_{i}, \hat{A}_{j}\right\}=C_{i j k} \hat{A}_{k} .
\end{aligned}
$$

(We are using the same notation for operators and c-number functions in the phase space since it will not create any confusion.) Using the Lie-Baxter algebras as in the quantum case [4], we can introduce the tilde variables in the phase space as

$$
\hat{A}_{i}=A_{i}-\tilde{A}_{i} \text {. }
$$


Therefore, the tilde and non-tilde variables satisfy the following relations:

$$
\begin{aligned}
& \left\{A_{i}, \tilde{A}_{j}\right\}=0, \\
& \left\{A_{i}, A_{j}\right\}=C_{i j k} A_{k}, \\
& \left\{\tilde{A}_{i}, \tilde{A}_{j}\right\}=-C_{i j k} \tilde{A}_{k} .
\end{aligned}
$$

Representations of Lie groups through the Poisson brackets are, in general, projective representations. That is, given a symmetry group characterized by $A_{i} \diamond A_{j}=$ $C_{i j k} A_{k}$, the realization in terms of Poisson brackets is given by

$$
\left\{A_{i}, A_{j}\right\}=C_{i j k} A_{k}+d_{i j},
$$

where $d_{i j}$ are pure numbers [18]. In this situation, using the relations $\left\{A_{i}, A_{j}\right\}=$ $C_{i j k} A_{k}+d_{i j},\left\{\tilde{A}_{i}, \tilde{A}_{j}\right\}=-C_{i j k} \tilde{A}_{k}-d_{i j}$ and the approach of Ref. [4], the thermoalgebra is modified to assume the following relations

$$
\begin{aligned}
& \left\{A_{i}, A_{j}\right\}=C_{i j k} A_{k}+d_{i j}, \\
& \left\{A_{i}, \hat{A}_{j}\right\}=C_{i j k} A_{k}+d_{i j}, \\
& \left\{\hat{A}_{i}, \hat{A}_{j}\right\}=C_{i j k} \hat{A}_{k} .
\end{aligned}
$$

Note that the hat-functions defining a subalgebra of the thermoalgebra is still a faithful representation of the original Lie-symmetry.

The generators of symmetries acting on the dynamical variables are defined by the following relation

$$
\bar{A}(\lambda)=e^{-\lambda\{\hat{D},\}} \bar{A}(\lambda=0),
$$

where $\bar{A}$ stands for the dynamical variables $A, \hat{A}$ or $\tilde{A}$ (this notation will be used throughout this paper); and, $\hat{D}$ is a general generator defining a one parameter $(\lambda)$ subgroup. In particular, if $\lambda$ is the time parameter, we can derive from Eq. (17) the equation of motion

$$
\dot{\bar{A}}=\{\bar{A}, \hat{H}\} .
$$

When $\bar{A}=A$, Eq. (18) reduces to $\dot{A}=\{A, H\}$, and the usual classical formalism is obtained.

An explicit form for the Poisson bracket in Eq. (18) is

$$
\{A, B\}=\sum_{a=1}^{2}\left(\frac{\partial A}{\partial x_{a}} \frac{\partial B}{\partial p_{a}}-\frac{\partial A}{\partial p_{a}} \frac{\partial B}{\partial x_{a}}\right),
$$


where $x_{1}=q, x_{2}=-\tilde{q}, p_{1}=p$ and $p_{2}=\tilde{p}$. Then we have a pair of Hamilton equations:

$$
\begin{aligned}
& \dot{x}_{a}=\left\{x_{a}, \hat{H}\right\}=\frac{\partial \hat{H}}{\partial p_{a}}, \\
& \dot{p}_{a}=\left\{p_{a}, \hat{H}\right\}=-\frac{\partial \hat{H}}{\partial x_{a}},
\end{aligned}
$$

where $a=1,2$. These equations will be used in Section 6 in connection with the $S U(1,1)$ group and dissipative systems.

\section{CLASSICAL UNITARY REPRESENTATIONS FOR THE THERMAL GALILEI GROUP}

Following Eqs. (4)-(6), we write the thermal Galilei algebra (denoted by $\mathscr{G}_{T}$ ) for a classical system as

$$
\begin{aligned}
{\left[J_{i}, J_{j}\right] } & =0, \quad\left[\hat{J}_{i}, \hat{J}_{j}\right]=i \varepsilon_{i j k} \hat{J}_{k}, \\
{\left[J_{i}, P_{j}\right] } & =0, \quad\left[\hat{J}_{i}, \hat{P}_{j}\right]=i \varepsilon_{i j k} \hat{P}_{k}, \\
{\left[J_{i}, K_{j}\right] } & =0, \quad\left[\hat{J}_{i}, \hat{K}_{j}\right]=i \varepsilon_{i j k} \hat{K}_{k}, \\
{\left[K_{i}, H\right] } & =0, \quad\left[\hat{K}_{i}, \hat{H}\right]=i \hat{P}_{i}, \\
{\left[P_{i}, K_{j}\right] } & =0, \quad\left[\hat{P}_{i}, \hat{K}_{j}\right]=0 \\
{\left[\hat{J}_{i}, J_{j}\right] } & =\left[J_{i}, \hat{J}_{j}\right]=i \varepsilon_{i j k} J_{k}, \\
{\left[\hat{J}_{i}, P_{j}\right] } & =\left[J_{i}, \hat{P}_{j}\right]=i \varepsilon_{i j k} P_{k}, \\
{\left[\hat{J}_{i}, K_{j}\right] } & =\left[J_{i}, \hat{K}_{j}\right]=i \varepsilon_{i j k} K_{k}, \\
{\left[\hat{K}_{i}, H\right] } & =\left[K_{i}, \hat{H}\right]=i P_{i}, \\
{\left[\hat{P}_{i}, K_{j}\right] } & =\left[P_{i}, \hat{K_{j}}\right]=-i M \delta_{i j},
\end{aligned}
$$

(the other commutation relations are null), where $\varepsilon_{i j k}$ is the Levi-Cevita tensor, $i, j, k=1,2,3$, and the generators of the symmetries are $\hat{P}$ for translations, $\hat{J}$ for rotations, $\hat{K}$ for the Galilei boost and $\hat{H}$ for the time translations. These operators from a subalgebra of $\mathscr{G}_{T}$, called $\hat{\mathscr{G}}$, which is manifestly a faithful representation of the Galilei Group. Moreover, the $\mathscr{G}$-algebra of the dynamical observables (non-hat operators) is abelian. 
In Eq. (22), $\hat{M}=0$ whilst $M$ is a constant operator, that is $M=m \mathbf{1}$. In order to define the Galilei transformation for a point mass, $m$ is considered real and different from zero. Thus let the operator of position $(Q)$ be defined by

$$
K=m Q-t P .
$$

Notice that this relation among $K, P$ and $Q$ is similar to that used in quantum mechanics, but here $Q$ and $P$ commute with one another.

A representation where $P$ and $Q$ are diagonal is specified by: $P|p, q\rangle=$ $p|p, q\rangle, Q|p, q\rangle=q|p, q\rangle$, so that $\langle q, p \mid \theta\rangle=\theta(p, q)$ is a vector of the representation space, which is a Hilbert space, say $\mathscr{H}$, on the phase space of the $(q, p)$ points. Then, the other operators of $\mathscr{G}_{T}$ can be written as

$$
\begin{aligned}
& J_{i}=L_{i}+S_{i}, \\
& H=\frac{P^{2}}{2 m}+C_{1}, \\
& \hat{J}_{i}=\hat{L}_{i}+\hat{S}_{i}, \\
& \hat{K}_{i}=i m \frac{\partial}{\partial P_{i}}+i t \frac{\partial}{\partial Q_{i}}, \\
& \hat{P}_{i}=-i \frac{\partial}{\partial Q_{i}}, \\
& \hat{H}=i \frac{\partial}{\partial t},
\end{aligned}
$$

where

$$
\begin{aligned}
& \hat{L}_{i}=i \varepsilon_{i j k}\left(Q_{k} \frac{\partial}{\partial Q_{j}}+P_{k} \frac{\partial}{\partial P_{j}}\right), \\
& L_{i}=\varepsilon_{i j k} Q_{j} P_{k},
\end{aligned}
$$

$\hat{S}_{i}$ are the spin operators (a representation of $S O(3)$ such that $\widetilde{S}$ commutes with every operator defined on the space $(p, q))$, and $C_{1}$ is a Casimir invariant as discussed later in this section.

The operators $P$ and $Q$ can be interpreted as the momentum and position operators, since they satisfy the Galilei boost conditions, namely

$$
\langle\theta|\exp (-i v \hat{K}) Q \exp (i v \hat{K})| \phi\rangle=\langle\theta|Q| \phi\rangle+v t\langle\theta \mid \phi\rangle,
$$

and

$$
\langle\theta|\exp (-i v \hat{K}) P \exp (i v \hat{K})| \phi\rangle=\langle\theta|P| \phi\rangle+m v\langle\theta \mid \phi\rangle,
$$


where $|\theta\rangle$ and $|\phi\rangle(\in \mathscr{H})$ are arbitrary states of the system. Besides, $\hat{P}$ is the generator for spatial translation, for $\left[\hat{P}_{i}, Q_{j}\right]=-i \delta_{i j}$. Then, $L$ is the angular momentum, and $H$ is the Hamiltonian.

In the case when $\hat{S}_{i}=S_{i}=0, \mathscr{G}_{T}$ has two non-null invariants, which have fixed values within the irreducible representation, given by

$$
\begin{aligned}
C_{1} & =\frac{P^{2}}{2 m}-H, \\
C_{2} & =\frac{\hat{P}}{m} P-\hat{H} .
\end{aligned}
$$

Following the case of the Galilean quantum mechanics [28, 29], the expectation value of a dynamical variable $\bar{A}$ in a state $|\theta\rangle$ is defined by

$$
\langle\bar{A}\rangle=\langle\theta|\bar{A}| \theta\rangle \text {. }
$$

On the other hand, the temporal evolution of $\bar{A}$ is given by

$$
\left\langle\theta_{0}\right| \exp (i t \hat{H}) \bar{A} \exp \left((-i t \hat{H})\left|\theta_{0}\right\rangle=\left\langle\theta_{0}|\bar{A}(t)| \theta_{0}\right\rangle .\right.
$$

Therefore, we have defined a Heisenberg picture for the temporal evolution of the dynamical variables, and from Eq. (37) we obtain

$$
i \partial_{t} \bar{A}=[\bar{A}, \hat{H}] \text {. }
$$

Using Eq. (35), Eq. (38) is written as

$$
i \partial_{t} \bar{A}=\left[\bar{A}, \frac{\hat{P}}{m} P\right],
$$

which shows that the value of $C_{2}$ does not play a special role in this case (as it can be seen later, in fact $C_{2}=0$ ).

In the Schrödinger picture, we derive the following equation for the evolution of the state

$$
i \partial_{t}|\theta(t)\rangle=\hat{H}|\theta(t)\rangle
$$

where $\hat{H}$ is given by Eq. (35), and $f(q, p ; t)=|\theta(q, p ; t)|^{2}$ is defined as the distribution function in the phase space. Writing $\theta(q, p)=\psi(t) \phi(q, p)$, Eq. (39) gives rise to the following eigenvalue equation

$$
\hat{H} \phi(q, p)=v \phi(q, p) \quad \text { and } \quad \psi(t)=\psi(0) e^{-i v t} .
$$

Notice that by the definition of the thermoalgebra, to every non-hat operator ( say $A$ ) there is a hat-operator (say $\hat{A}$ ). This is a one-to-one mapping, which may be determined using the explicit representation of the thermoalgebra given by 
Eqs. (23)-(29). Then, we can define the one-to-one hat-mapping, $h: A \rightarrow \hat{A}$, such that $\hat{A}=i\{A$,$\} , where \{$,$\} is the Poisson brackets given by$

$$
\hat{A}=i\{A,\}=i \frac{\partial A}{\partial q} \frac{\partial}{\partial p}-i \frac{\partial A}{\partial p} \frac{\partial}{\partial q} .
$$

We have, hence, the following correspondence for the Lie algebra elements:

$$
\begin{aligned}
P & \rightarrow \hat{P}=i\{P,\}, \\
K & \rightarrow \hat{K}=i\{K,\}, \\
L & \rightarrow \hat{L}=i\{L,\}, \\
H & \rightarrow \hat{H}=i\{H,\}, \\
M & \rightarrow \hat{M}=i\{M,\}=0 .
\end{aligned}
$$

As a consequence, the thermoalgebra reduces to a secondary Lie algebra, which is a type of extension of the adjoint representation of a given (primary) Lie algebra [16]. Therefore, $\hat{H}$ is the classical Liouvillian operator and Eq. (39) is the Schönberg-Liouville wave equation [19]. Indeed, we have derived the classical mechanics since $f(q, p)=|\theta(q, p)|^{2}$ is a solution of Eq. (39), and the average given by Eq. (36) reduces to the usual average in the phase space for the non-hat operators, that is

$$
\begin{aligned}
\langle A\rangle & =\langle\theta|A| \theta\rangle=\int d p d q \theta(q, p) A(q, p) \theta(q, p) \\
& =\int d p d q f(q, p) A(q, p) .
\end{aligned}
$$

The hat-mapping $(h)$ and the thermoalgebra defined by Eqs. (4)-(6) satisfy the following properties (such properties are the classical counterpart of the quantum case [4]):

P.1. $h$ is a linear mapping: $\left(A_{1}+a A_{2}\right)^{\wedge}=\hat{A}_{1}+a \hat{A}_{2}$.

P.2. $h$ is a derivation: $\left(\prod_{i=1}^{n} A_{i}\right)^{\wedge}=\sum_{i=1}^{n}\left(A_{1} \cdots A_{i-1} \hat{A}_{i} A_{i+1} \cdots A_{n}\right)$.

Proposition 1. Given $C \in \mathscr{G}$ and $\{C, A\}=0$, for every $A \in \mathscr{G}$, then $C$ and $\hat{C}$ are two Casimir invariants of $\mathscr{G}_{T}$.

Since we know the invariants of the Galilei group, considering Proposition 1, we can write the Casimir invariants of $\mathscr{G}_{T}$ in the general situation in which $S \neq 0$. These invariants are $C_{1}$ and $C_{2}$, given by the Eq. (34) and (35), respectively, and

$$
\begin{aligned}
& C_{3}=(J-L)^{2}, \\
& C_{4}=\left[(J-L)^{2}\right]^{\wedge} .
\end{aligned}
$$


Both $C_{4}=\hat{C}_{3}$ and $C_{2}=\hat{C}_{1}$ have a fixed value in an irreducible representation. But in accordance with the properties of the hat-mapping $C_{2}$ and $C_{4}$ are null constants.

With the properties P.1 and P.2, and the Proposition 1, we can follow closely the Loinger's work (which is based directly on the structure of the Poisson brackets) [11] to conclude that when $M=0$ in Eq. (22), we cannot find a physical representation for $\mathscr{G}_{T}$.

Similar to the quantum TFD, we can introduce in this classical formalism an arbitrary parameter $\alpha$ to be specified [8]. Indeed, the most general form of Eq. (36) is

$$
\begin{aligned}
\langle\bar{A}\rangle & =\left\langle f^{\alpha}|\bar{A}| f^{1-\alpha}\right\rangle \\
& =\int d p d q f^{\alpha}(q, p) A(q, p) f^{1-\alpha}(q, p) .
\end{aligned}
$$

Then, Eq. (36) is obtained when $\alpha=\frac{1}{2}$. As is the case in TFD, however, there are other possibilities for $\alpha$. For instance, if $\alpha=0$ and the basis of the states is such that the operators $P$ and $\hat{P}$ are diagonal, we obtain the formalism called dynamics of correlations [30, 31]. In fact, if $P$ and $\hat{P}$ are c-number operators, then $P|p, k\rangle=$ $p|p, k\rangle, \hat{P}|p, k\rangle=k|p, k\rangle$, so that $\langle p, k \mid f\rangle=f_{k}(p)$. As a consequence

$$
\begin{aligned}
f_{k}(p) & =\int d p^{\prime} d q\left\langle p, k \mid p^{\prime}, q\right\rangle f\left(p^{\prime}, q\right) \\
& =\int d q e^{i k p} f(q, p) .
\end{aligned}
$$

The $f_{k}(p)$-functions are the correlation patterns in the dynamics of correlations $[30,31]$.

Closing this section, let us write the average, Eq. (48), for the thermal equilibrium:

$$
\langle\bar{A}\rangle=\left\langle f^{\alpha}|\bar{A}| f^{1-\alpha}\right\rangle=\int d p d q e^{-\alpha(\beta / 2) H} \bar{A} e^{(\alpha-1)(\beta / 2) H},
$$

still a similar case to that in TFD.

\section{SCHÖNBERG-LIOUVILLE WAVE EQUATION FOR THE OSCILLATOR SYSTEM}

It is shown in the previous section that, similar to TFD, the dynamics of a system is generated by hat operators, which are in correspondence with the original dynamical variables via the Poisson brackets:

$$
\begin{array}{cc}
\text { Quantum TFD } & \text { Liouvillian Systems } \\
\hat{A}=A-\tilde{A}, \quad \hat{A}=i\{A,\}=i\left(\frac{\partial A}{\partial q} \frac{\partial}{\partial_{p}}-\frac{\partial A}{\partial p} \frac{\partial}{\partial_{q}}\right) .
\end{array}
$$


This relationship between the classical operators and quantum operators is not transparent. We would like to see if there are additional connections. In order to do this, we shall first represent the $p$-variable in its Fourier representation, such that

$$
p \rightarrow i \partial_{\tilde{q}} \quad \text { and } \quad \partial_{p} \rightarrow i \tilde{q} .
$$

$\hat{p}=i m \partial_{q}$ does not change. In particular, the generator of rotations is given by

$$
\hat{L}=i \varepsilon_{i j k}\left(q_{k} \partial_{q_{j}}+p_{k} \partial_{p_{j}}\right) \rightarrow i \varepsilon_{i j k}\left(q_{k} \partial_{q_{j}}-\tilde{q}_{j} \partial_{\tilde{q}_{k}}\right)=i \varepsilon_{i j k}\left(q_{k} \partial_{q_{j}}+\tilde{q}_{k} \partial_{\tilde{q}_{j}}\right) .
$$

Assuming that $H=T+V$, we get

$$
\hat{H}=-i\left(p \partial_{q}-\partial_{q} V \partial_{p}\right) \rightarrow\left(\partial_{q} \partial_{\tilde{q}}-\partial_{q} V \tilde{q}\right) .
$$

For a harmonic oscillator, $\hat{H}=-i\left(p \partial_{q}-q \partial_{p}\right)$ resembles the quantum angular momentum operator $L_{z}$, which can be considered as $L_{z}=a^{\dagger} a-\tilde{a}^{\dagger} \tilde{a}$, where $a$ and $\tilde{a}$ are two independent annihilation operators. We will show in the following that this is indeed the case.

Consider a rotation of the form

$$
\left(\begin{array}{c}
q \\
\tilde{q}
\end{array}\right)=\left(\begin{array}{cc}
u & v \\
-v & u
\end{array}\right)\left(\begin{array}{c}
X \\
\tilde{X}
\end{array}\right), \quad\left(\begin{array}{c}
X \\
\tilde{X}
\end{array}\right)=\left(\begin{array}{cc}
u & -v \\
v & u
\end{array}\right)\left(\begin{array}{c}
q \\
\tilde{q}
\end{array}\right),
$$

with $u^{2}=v^{2}=1 / 2, \hat{p} \rightarrow i m u\left(\partial_{X}+\partial_{\tilde{X}}\right)$. For a system of harmonic oscillators we have

$$
\begin{aligned}
\hat{H} & =\frac{1}{2} \sum_{i}\left[-\partial_{X_{i}}^{2}+X_{i}^{2}+\partial_{\tilde{X}_{i}}^{2}-\tilde{X}_{i}^{2}\right] \\
& =H(X)-H(\tilde{X}),
\end{aligned}
$$

which is remarkably similar to the one in the TFD formalism. A more general system would then become

$$
\hat{H}=\sum_{i}\left[-\frac{1}{2}\left(\partial_{X_{i}}^{2}-\partial_{\tilde{X}_{i}}^{2}\right)+\frac{1}{\sqrt{2}}\left(X_{i}-\tilde{X}_{i}\right) V_{i}(X+\tilde{X})\right],
$$

where $V_{i} \equiv \partial_{q_{i}} V$. Even for such a general system the time generator for the distribution function is anti-tilde-invariant in the TFD sense.

To see more connections with TFD, consider the following transformation

$$
a=\frac{1}{\sqrt{2}}\left(X+\partial_{X}\right), \quad a^{\dagger}=\frac{1}{\sqrt{2}}\left(X-\partial_{X}\right) .
$$

The original Hamiltonian becomes

$$
\begin{aligned}
H & =\frac{1}{2}\left[p^{2}+q^{2}\right]=\frac{1}{2}\left[q^{2}-\partial_{\tilde{q}}^{2}\right]=\frac{1}{4}\left[(X+\widetilde{X})^{2}-\left(\partial_{X}-\partial_{\tilde{X}}\right)^{2}\right] \\
& =g_{1}+g_{3},
\end{aligned}
$$


where $g_{1}$ and $g_{3}$ are two of the three generators defined in TFD as $g_{1}=a \tilde{a}+a^{\dagger} \tilde{a}^{\dagger}$, $g_{2}=i\left(a \tilde{a}-\tilde{a}^{\dagger} a^{\dagger}\right)$, and $g_{3}=a^{\dagger} a+\tilde{a}^{\dagger} \tilde{a}+1[7,8]$. They commute with the time generator $\tilde{H}$. If a state $|0\rangle$ satisfies the Schönberg-Liouville equation, so does $e^{\alpha H}|0\rangle$ where $\alpha$ is an arbitrary c-number, since $[H, \hat{H}]=0$. In fact, $f(H)|0\rangle$ (where $f(H)$ is an arbitrary function of $H$ ) is also a valid solution in the classical case. Henceforth, we have the following correspondence between the Liouville system and TFD:

$$
\begin{aligned}
& \text { Liouvillian System Thermofield Dynamics } \\
& \hat{H}=i\{H, .\} \quad \rightarrow \hat{H}=H-\tilde{H}, \\
& H \rightarrow G \text { Transformation Generators, } \\
& |\theta\rangle \quad|0\rangle .
\end{aligned}
$$

The last correspondence is the same for the quantum system as well.

Consider now that, the vacuum state of the quantum system is given by

$$
\psi_{0}=e^{-1 / 2\left(X^{2}+\tilde{X}^{2}\right)}=e^{-1 / 2\left(x^{2}+y^{2}\right)}=\int d p e^{i p y} e^{-H(x, p)}
$$

where $H(x, p)=\frac{1}{2}\left(x^{2}+p^{2}\right)$. This solution corresponds to a classical system in thermal equilibrium at $\beta=1$. The obvious solutions of the classical Liouville equation are

$$
e^{\alpha H} \psi_{0}=\int d p e^{i p y} e^{-(1+\alpha) 1 / 2\left(x^{2}+p^{2}\right)} .
$$

The following two generators

$$
a \tilde{a} \pm a^{\dagger} \tilde{a}^{\dagger} \rightarrow\left\{\begin{array}{l}
q^{2}+p^{2}+\partial_{q}^{2}+\partial_{p}^{2} \\
q \partial_{q}+p \partial_{p}
\end{array},\right.
$$

however, do not generate any new class of solutions. The obvious solution is obtained by the use of polar coordinates $p=r \cos \theta, q=r \sin \theta, \hat{L} \rightarrow \partial_{\theta}$. A time invariant solution must be of form $f\left(r^{2}\right)=f\left(p^{2}+x^{2}\right)$ that is independent of $\theta$.

In TFD, the canonical transformations are, as a matter of fact, a restricted class of transformations. If we write [8]

$$
e^{-G}\left(\begin{array}{c}
a \\
\tilde{a}^{\dagger}
\end{array}\right) e^{G}=\left(\begin{array}{cc}
B_{11} & B_{12} \\
B_{21} & B_{22}
\end{array}\right)\left(\begin{array}{c}
a \\
\tilde{a}^{\dagger}
\end{array}\right),
$$

then we have the constraint that $B_{12} B_{21}=\left\langle 0\left|a^{\dagger} a\right| 0\right\rangle$ be positive. A close examination reveals that the solution $e^{\alpha H} \psi_{0}$ with real $\alpha$ is actually forbidden in the quantum case. However there is no such constraint in the classical system. Therefore any solution $f\left(x^{2}+p^{2}\right)$ is a valid solution in the classical case. 


\section{SCHÖNBERG-FOCK SPACE AND REPRESENTATIONS FOR THE THERMAL GALILEI GROUP}

In Section 2 the unitary representation for the thermal Galilei algebra, $\mathscr{G}_{T}$, has been studied, where the representation space was the Hilbert space denoted by $\mathscr{H}$. In this Section, a unitary representation for $\mathscr{G}_{T}$ is derived using the Fock space $(\mathscr{F})$ defined by the tensor product of $\mathscr{H}$; that is

$$
\mathscr{F}=\underset{m}{\oplus} \mathscr{H}^{\otimes m}, \quad m=0,1,2, \ldots
$$

The elements of $\mathscr{G}_{T}$ are assumed to be defined in $\mathscr{F}$, which will be called Schönberg-Fock space.

Let $\psi(q, p)$ and $\psi^{\dagger}(q, p)$ be the basic field operators satisfying the commutation relations

$$
\begin{aligned}
{\left[\psi(q, p), \psi^{\dagger}\left(q^{\prime}, p^{\prime}\right)\right]_{ \pm} } & =\delta\left(q-q^{\prime}\right) \delta\left(p-p^{\prime}\right), \\
{\left[\psi(q, p), \psi\left(q^{\prime}, p^{\prime}\right)\right]_{ \pm} } & =\left[\psi^{\dagger}(q, p), \psi^{\dagger}\left(q^{\prime}, p^{\prime}\right)\right]_{ \pm}=0,
\end{aligned}
$$

where $-(+)$ in the brackets defines the classical bosons (fermions). For the sake of simplicity, we use in this section the notation: $\tau=(p, q) ; \psi(\tau)=\psi(q, p)$; $\delta(\tau)=\delta\left(q-q^{\prime}\right) \delta\left(p-p^{\prime}\right)$; we also assume that the particle spin is $S_{i}=0$.

In the space $\mathscr{F}$ there is a state $|0\rangle$, such that $\psi(\tau)|0\rangle=0,\langle 0 \mid 0\rangle=1$, and $\psi^{\dagger}\left(\tau_{i}\right)|0\rangle=\left|\chi_{i}\right\rangle$. A general vector in $\mathscr{F}$ is defined by

$$
|\chi(t)\rangle=\theta(t)_{0}|0\rangle+\sum_{i=1}^{\infty} \frac{1}{\sqrt{n !}} \int \theta\left(\tau_{1}, \tau_{2}, \ldots, \tau_{n} ; t\right)\left|\chi_{n}\right\rangle d^{n} \tau,
$$

where $d^{n} \tau=d \tau_{1} d \tau_{2} \cdots d \tau_{n},\left|\chi_{n}\right\rangle=\psi^{\dagger}\left(\tau_{1}\right) \psi^{\dagger}\left(\tau_{2}\right) \cdots \psi^{\dagger}\left(\tau_{n}\right)|0\rangle$, and $\theta\left(\tau_{1}, \tau_{2}, \ldots, \tau_{n} ; t\right)$ are symmetric or anti-symmetric functions of the Hilbert space $\mathscr{H}$, such that

$$
\theta\left(\tau_{1}, \tau_{2}, \ldots, \tau_{n} ; t\right)=\frac{1}{\sqrt{n !}}\left\langle\chi_{n} \mid \chi\right\rangle=\frac{1}{\sqrt{n !}}\left\langle 0\left|\prod_{i=1}^{n} \psi\left(\tau_{i}\right)\right| \chi\right\rangle .
$$

Besides

$$
\left\langle\chi \mid \chi^{\prime}\right\rangle=\theta_{0}^{*} \theta_{0}^{\prime}+\sum_{i=1}^{\infty} \int \theta^{*}\left(\tau_{1}, \tau_{2}, \ldots, \tau_{n} ; t\right) \theta^{\prime}\left(\tau_{1}, \tau_{2}, \ldots, \tau_{n} ; t\right) d^{n} \tau .
$$

Therefore, for each operator $\bar{A}\left(\tau_{1}, \tau_{2}, \ldots, \tau_{n}\right)$ defined on $\mathscr{H}$, and depending symmetrically on the variables $\tau_{1}, \tau_{2}, \ldots, \tau_{n}$, we can introduce a correspondent operator acting on $\mathscr{F}$ in the following way

$$
\overline{\mathscr{A}}=\frac{1}{n !} \int \psi^{\dagger}\left(\tau_{1}\right) \psi^{\dagger}\left(\tau_{2}\right) \cdots \psi^{\dagger}\left(\tau_{n}\right) \bar{A}\left(\tau_{1}, \tau_{2}, \ldots, \tau_{n}\right) \psi\left(\tau_{n}\right), \ldots, \psi\left(\tau_{2}\right) \psi\left(\tau_{1}\right) d^{n} \tau .
$$


A representation for the Galilei thermoalgebra is then obtained with such a kind of operators when $n=1$. Then, using Eqs. (23-29) and $n=1$ in Eq. (57), the nonnull commutation relations for the thermal Galilei group are now given by

$$
\begin{aligned}
& {\left[\hat{\mathscr{J}}_{i}, \hat{\mathscr{J}}_{j}\right]=i \varepsilon_{i j k} \hat{\mathscr{J}}_{k},} \\
& {\left[\hat{\mathscr{J}}_{i}, \hat{\mathscr{P}}_{j}\right]=i \varepsilon_{i j k} \hat{\mathscr{P}}_{k},} \\
& {\left[\hat{\mathscr{J}}_{i}, \hat{\mathscr{K}}_{j}\right]=i \varepsilon_{i j k} \hat{\mathscr{K}}_{k} \text {, }} \\
& {\left[\hat{\mathscr{K}}_{i}, \hat{\mathscr{H}}\right]=i \hat{\mathscr{P}}_{i},} \\
& {\left[\hat{\mathscr{J}}_{i}, \mathscr{J}_{j}\right]=\left[\mathscr{J}_{i}, \hat{\mathscr{J}}_{j}\right]=i \varepsilon_{i j k} \mathscr{J}_{k},} \\
& {\left[\hat{\mathscr{J}}_{i}, \mathscr{P}_{j}\right]=\left[\mathscr{\mathscr { J }}_{i}, \hat{\mathscr{P}}_{j}\right]=i \varepsilon_{i j k} \mathscr{P}_{k},} \\
& {\left[\hat{\mathscr{J}}_{i}, \mathscr{K}_{j}\right]=\left[\mathscr{J}_{i}, \hat{\mathscr{K}}_{j}\right]=i \varepsilon_{i j k} \mathscr{K}_{k} \text {, }} \\
& {\left[\hat{\mathscr{K}}_{i}, \mathscr{H}\right]=\left[\mathscr{K}_{i}, \hat{\mathscr{H}}\right]=i \mathscr{P}_{i},} \\
& {\left[\hat{\mathscr{P}}_{i}, \mathscr{K}_{j}\right]=\left[\mathscr{P}_{i}, \hat{\mathscr{K}}_{j}\right]=-i \mathscr{N} m \delta_{i j} .}
\end{aligned}
$$

$\mathscr{N}$, the number operator, is defined by

$$
\mathscr{N}=\int \mathscr{N}(\tau) d \tau=\int \psi^{\dagger}(\tau) \psi(\tau) d \tau
$$

such that

$$
\mathscr{N}\left|\chi_{n}\right\rangle=n\left|\chi_{n}\right\rangle \text {. }
$$

Following the method given in Section 3 , a dynamical variable $\overline{\mathscr{A}}$ has its average in a state $|\chi\rangle$ defined by

$$
\langle\overline{\mathscr{A}}\rangle=\langle\chi|\overline{\mathscr{A}}| \chi\rangle
$$

On the other hand, the temporal evolution of $\overline{\mathscr{A}}$ is given by

$$
\left\langle\chi_{0}|\exp (i t \hat{\mathscr{H}}) \overline{\mathscr{A}} \exp (-i t \hat{\mathscr{H}})| \chi_{0}\right\rangle=\left\langle\chi_{0}|\overline{\mathscr{A}}(t)| \chi_{0}\right\rangle \text {. }
$$

Thus, we have defined a Heisenberg picture for the temporal evolution of the dynamical variables, and from Eq. (61) we obtain

$$
i \partial_{t} \overline{\mathscr{A}}=[\overline{\mathscr{A}}, \hat{\mathscr{H}}] .
$$

In the Schrödinger picture, the equation for the evolution of the state is

$$
i \partial_{t}|\chi(t)\rangle=\hat{\mathscr{H}}|\chi(t)\rangle .
$$


Using Eq. (63), we can show that

$$
i \partial_{t} \theta\left(\tau_{1}, \tau_{2}, \ldots, \tau_{n} ; t\right)=\hat{H}\left(\tau_{1}, \tau_{2}, \ldots, \tau_{n}\right) \theta\left(\tau_{1}, \tau_{2}, \ldots, \tau_{n} ; t\right),
$$

where $\hat{H}$ is the Liouvillian for $n$ particles. To get a physically consistent interpretation of the theory we define $f\left(\tau_{1}, \tau_{2}, \ldots, \tau_{n} ; t\right)=\left|\theta\left(\tau_{1}, \tau_{2}, \ldots, \tau_{n} ; t\right)\right|^{2}$ as the $n$-particle distribution function in the phase space of $(p, q)$.

This second quantization formalism in the Fock space of symmetric or anti-symmetric classical states was first proposed by Schönberg [19]. However, we would like to emphasize here that the structure of classical statistical mechanics, in particular the concept of phase space, Poisson bracket and Liouville equation for $n$ particles, has emerged naturally from the thermoalgebra structure; this aspect of consistency is not present in the usual methods related to the Schönberg approach [19-23].

\section{REPRESENTATIONS OF $S U(1,1)$ AND THE THERMAL CLASSICAL OSCILLATOR}

Consider the double phase space defined in Section 2, where the Poisson brackets for the tilde-non-tilde variables are specified by Eq. (19). A realization of the rotation group in this double phase space is defined by the relation

$$
\left\{L_{i}, L_{j}\right\}=\varepsilon_{i j k} L_{k},
$$

such that each component of $L$ is a function of $\left(x_{a}, p_{b}\right), a, b=1,2$. The Casimir invariant is

$$
\mathscr{C}^{2}={L_{1}}^{2}+{L_{2}}^{2}+{L_{3}}^{2}
$$

and we suppose a system described by the following hat Hamiltonian (the generator of time translation)

$$
\hat{H}=N \mathscr{C}+M L_{2},
$$

where $N$ and $M$ are constants. Let us introduce the following set of variables $L_{1}=$ $(i / 2)\left(L_{-}+L_{+}\right)$and $L_{2}=\frac{1}{2}\left(L_{+}-L_{-}\right)$( or $\left.L_{ \pm}=\mp\left(L_{2} \pm i L_{1}\right)\right)$. The algebra of $L_{+}$, $L_{-}$and $L_{3}$ is a representation of $S U(1,1)$ for

$$
\begin{gathered}
\frac{1}{i}\left\{L_{ \pm}, L_{3}\right\}=\mp L_{ \pm}, \\
\frac{1}{i}\left\{L_{-}, L_{+}\right\}=-2 L_{3} .
\end{gathered}
$$


In terms of $L_{+}, L_{-}$and $L_{3}, \mathscr{C}$ is given by $\mathscr{C}^{2}=L_{3}{ }^{2}-L_{+} L_{-}$. Since we have an algebra of $S U(1,1)$, we can define an algebra for oscillator-like operators. That is, we define the variables $a$ and $b$, such that

$$
\begin{aligned}
L_{+} & =\frac{1}{2}\left(a^{2}-b^{2}\right), \\
L_{-} & =\frac{1}{2}\left(a^{* 2}-b^{* 2}\right), \\
L_{3} & =\frac{1}{2}\left(a a^{*}+b b^{*}\right),
\end{aligned}
$$

where $a^{*}\left(b^{*}\right)$ is the complex conjugate of $a(b)$. Then the algebra for the variables $a, b$ and their complex conjugates is given by the relations $\{a, b\}=0,\left\{a, a^{*}\right\}=i$, and $\left\{b, b^{*}\right\}=i$. The other relations are zero.

The basic variables $x_{a}, p_{a}$ of the double phase space specified by Eq. (19) can be introduced through a transformation defined by

$$
\begin{aligned}
& a=\left(\frac{1}{2 \Omega}\right)^{1 / 2}\left(p_{1}-i \Omega x_{1}\right), \\
& b=\left(\frac{1}{2 \Omega}\right)^{1 / 2}\left(p_{2}-i \Omega x_{2}\right),
\end{aligned}
$$

and the constants: $N=2 \Omega, M=-i \gamma, \Omega^{2}=k-(\gamma / 2 m)^{2}$. Then, the Hamiltonian, Eq. (67), is written as

$$
\hat{H}=\frac{1}{2} p_{1} p_{2}+\frac{1}{2} \Omega^{2} x_{1} x_{2}+\frac{\gamma}{4}\left(p_{2} x_{2}-p_{1} x_{1}\right) .
$$

Using Eq. (75) and the Hamilton equations defined in section 2 by Eqs. (20) and (21), we have the following equations

$$
\begin{aligned}
& m \ddot{x}_{1}+\gamma \dot{x}_{1}+k x_{1}=0, \\
& m \ddot{x}_{2}-\gamma \dot{x}_{2}+k x_{2}=0 .
\end{aligned}
$$

These two equations describe a system of two oscillators: one is the usual dissipative harmonic oscillator, Eq. (76), and the other, Eq. (77), is a growingoscillator to which the energy of the dissipative oscillator flows. Such a classical dissipative system with two degrees of freedom was analysed by Feshbach and Tikochinsky [24], and has been recently studied in connection with quantum optics and thermal field theories [25-27].

\section{CONCLUSIONS}

In summary, the concept of thermal Lie algebra, which was originally developed in connection with the thermal quantum field theory, has been extended to the classical mechanics. Two types of representations for classical systems have been 
analyzed: (i) the unitary representations, where the representation space is a Hilbert space; (ii) the non-unitary representation, where the Lie product of the thermoalgebra is the Poisson brackets. In both cases the basic elements of the thermofield dynamics (TFD) approach could be imported to the classical mechanics.

In the case of unitary representations, we have obtained the Liouville equation and the Schönberg formalism for the classical statistical mechanics, which is the quantized version of classical systems assuming symmetric or antisymmetric states. Both, first-quantized and Fock-space version of the Liouville equation have been analyzed. The connections with the TFD formalism have been established, particularly by studying the example of a harmonic oscillator. In this case, it has been found that the classical harmonic oscillator is represented in a way almost identical to the quantum system. It is worth noting the fact that in such unitary representations, due to the concept of thermoalgebra, the thermal state of the system is defined as a pure (not mixed) state, in the sense that, it is is representated by vector in a Hilbert space. Besides, the concept of phase space, Poisson brackets and wave function in the phase space have emerged naturally in this approach.

In the case of non-unitary representations, a representation of the thermal $S U(1,1)$ algebra in a double phase space has been studied. The nature of the tilde observables is manifested through the form of the hat-Hamiltonian. As a consequence, we have obtained a system of two oscillators: one of which is the dissipative harmonic oscillator, and the other is a growing harmonic oscillator, the one that absorbs energy. We can see, therefore, that the role of the tilde variables is to introduce, in a non-obvious form, a growing oscillator to which the energy of the dissipative oscillator flows. This growing oscillator is, in this case, a simple model for the dissipative-oscillator environment, being that the effect of dissipation appears as a consequence of the structure of the hat-Hamiltonian.

\section{ACKNOWLEDGMENTS}

The work was supported in part by the Natural Sciences and Engineering Research Council of Canada, by the CAPES (a Brazilian Government Agency for Research), and by Materials Research Lab NSF-DMR-89-20538. The authors thank the referee for valuable suggestions.

\section{REFERENCES}

1. Preliminary results of this work have been presented at the "4th Workshop on Thermal Field Theory and Their Applications: Dalian-Thermo95, Dalian, China, 1995”; see A. Santana, F. C. Khanna, and H. Chu, Thermofield dynamics and classical systems, in "Proceedings, Dalian-Thermo 95” (Y. X. Gui, F. C. Khanna, and Z. B. Su, Eds.), p. 61, World Scientific, Singapore.

2. G. G. Emch, "Algebraic Methods in Statistical and Quantum Field Theory," Wiley, New York, 1972; W. Thirring, "Quantum Mechanics of Large Systems," Springer-Verlag, New York, 1983.

3. I. E. Segal, Ann. Math. 48 (1947), 930.

4. A. E. Santana and F. C. Khanna, Phys. Lett. A 203 (1995), 68.

5. E. J. Verboven, Phys. Lett. 21 (1966), 391; Physica 32 (1966), 2081. 
6. Y. Takahashi and H. Umezawa, Collective Phenomena 2 (1975), 55.

7. H. Umezawa, "Advanced Field Theory: Micro, Macro, and Thermal Physics," AIP, New York, 1993.

8. H. Chu and H. Umezawa, Int. J. Modern Phys. A 9 (1994), 2363.

9. A. E. Santana and F. C. Khanna, Lie groups and thermofield dynamics, in "Proceedings of the Dalian-THERMO95” (Y. X. Gui, F. C. Khanna, and Z. B. Su, Eds.), p. 55, World Scientific, Singapore.

10. B. O. Koopman, Proc. Natl. Acad. Sci. (USA) 17 (1931), 315.

11. A. Loinger, Ann. Phys. (N.Y.) 20 (1962), 132.

12. G. Lugarini and M. Pauri, Ann. Phys. (N.Y.) 44 (1967), 226.

13. T. Ali and E. Prugovečki, Physica A 89 (1977), 501.

14. C. George and I. Prigogine, Physica A 99 (1979), 369.

15. D. Bohm and B. J. Hiley, Found. Phys. 11 (1981), 179.

16. A. Santana, A. Matos Neto and J. D. M. Vianna, Hadronic J. 17 (1994), 539.

17. T. N. Sherry and E. C. G. Sudarshan, Phys. Rev. D 18 (1978), 4580.

18. E. C. G. Sudarshan and N. Mukunda, "Classical Dynamics: A Modern Perspective," Wiley, New York, 1974.

19. M. Schönberg, Il Nuovo Cimento 9 (1952), 1139; 10 (1953), 419; 10 (1953), 697.

20. A. E. Santana, A. Matos Neto, and J. M. Vianna, Int. J. Theoret. Phys. 28 (1989), 787.

21. M. Doi, J. Phys. A 9 (1976), 1465; 9 (1976), 1479.

22. R. Paul, "Field Theoretical Methods in Chemical Physics," Elsivier, Amsterdam, 1982.

23. A. Matos Neto and J. D. M. Vianna, Nuovo Cimento B 86 (1985), 117.

24. H. Feshbach and Y. Tikochinsky, Trans. N. Y. Acad. Sci., Ser. II 38 (1977), 44.

25. E. Celeghini, M. Rasetti, M. Tarlini, and G. Vitiello, Mod. Phys. Lett. B 3 (1989), 1213.

26. E. Celeghini, M. Rasetti, and G. Vitiello, Ann. Phys. (N.Y.) 215 (1992), 156.

27. Y. N. Srivastava, G. Vitiello, and A. Windon, Ann. Phys. (N.Y.) 238 (1995), 200.

28. E. Inönü and E. P. Wigner, Nuovo Cimento 9 (1952), 705.

29. M. Hamermesh, Ann. Phys. (N.Y.) 17 (1960), 518.

30. I. Prigogine, "Non-Equilibrium Statistical Mechanics," Wiley, New York, 1962.

31. I. Prigogine, C. George, F. Henin, and L. Rosenfeld, Chem. Scripta 4 (1973), 5. 